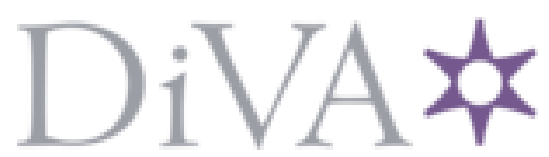

http://www.diva-portal.org

This is the published version of a paper presented at XXVe Congrès International de Linguistique et de Philologie Romanes, Innsbruck, 3 - 8 septembre 2007.

Citation for the original published paper:

Lindgren, C., Andersson, C. (2010)

Représentation de l'oral à l'écrit dans les traductions en français de livres suédois pour enfants. In: Iliescu, Maria; Siller-Runggaldier, Heidi M.; Danler, Paul (ed.), Actes du XXVe Congrès international de linguistique et de philologie romanes : Innsbruck 2007 (pp. 301-309). Walter de Gruyter

N.B. When citing this work, cite the original published paper.

Permanent link to this version:

http://urn.kb.se/resolve?urn=urn:nbn:se:du-15783 


\section{Représentation de l'oral à l'écrit dans les traductions en français de livres suédois pour enfants}

La traduction en français des livres pour enfants suédois met en jeu des différences linguistiques et culturelles importantes. Le suédois et le français manifestent évidemment plusieurs dissimilitudes au niveau lexical et grammatical, et, de plus, le sens même de la notion d'enfance et de celle de littérature pour enfants, et a fortiori les attentes sur le genre, sont distincts. ${ }^{1}$ De nos jours il apparait que, pour s'exprimer de façon extrême, les adultes suédois désirent rester très proches de l'enfance, alors que les enfants français ressemblent, du moins aux yeux des Suédois, à de petits adultes. Ceci transparait dans la culture en général et dans la littérature en particulier. Ce qui nous intéresse avant tout dans nos recherches traductologiques est la perspective de l'enfant: par quels moyens, à quel degré, est-ce que les auteurs et les traducteurs essaient de rendre la logique, la vision du monde, etc. des enfants. L'imitation de l'oral chez les personnages enfants est un des moyens favorisés par les auteurs pour se mettre au niveau de l'enfant. Cela dit, il convient de souligner que même les auteurs réputés pour faire parler leurs personnages comme de 〈vrais〉 enfants, comme par exemple Malika Ferdjoukh, ne visent pas forcément une reproduction exacte du language enfantin - ce qui mènerait certainement vers l'illisible, mais se servent de marqueurs linguistiques et typographiques pour créer une certaine illusion d'authenticité. Dans cet article nous allons présenter quelques choix orthographiques, lexicaux et grammaticaux qui contribuent, par leur rapprochement de l'écrit à l'oral, à rendre les personnages plus vrais, dans des livres suédois, et leur traitement dans les traductions françaises. L'analyse est basée sur un corpus de dix livres pour différents publics, des albums, des livres pour jeunes lecteurs et des livres pour lecteurs adolescents choisis parmi les 170 ouvrages traduits entre 1985 et $2005 .{ }^{2}$ Ce corpus

1 Pour ne citer que quelques exemples, Kåreland (1998; 2001), Rhedin (2004) ou Westin (1998) ont mis en valeur certaines particularités de la littérature suédoise pour enfants, notamment en comparaison avec la littérature française pour enfants.

2 Dans nos recherches antérieures (Lindgren et al. 2007; Renaud et al. 2007), nous avons pu constater que les livres pour enfants (moins de 15 ans) suédois ont été relativement bien reçus en France. L'âge d'or de la traduction se situe dans les années 1990. Dans la période 1985-2005, le nombre total de livres contemporains traduits pour la première fois s'élève à environ 170 . C'est surtout la maison d'édition L'école des loisirs qui a misé sur les livres pour enfants suédois, de même que Casterman. Flammarion, Albin Michel, Hachette et actuellement Thierry Magnier sont aussi représentés parmi les éditeurs les plus importants en ce domaine. Ces éditeurs ont un a priori favorable en faveur des livres pour enfants suédois et font leur choix selon des coups de cœur. 
a été complété par un corpus français contemporain de contrôle qui comprend six livres pour trois tranches d'âge.

En étudiant les traductions nous avons remarqué plusieurs tendances générales au niveau linguistique. Avant de présenter la représentation de l'oral à l'écrit dans ces traductions en français, nous allons dire quelques mots de trois tendances stylistiques que nous avons relevées, et qui ne sont pas sans influence sur la suite de notre exposé: la syntaxe, le vocabulaire, et la narration sont plus complexes dans la version française.

\section{Adaptation à des normes stylistiques en français}

\subsection{Une syntaxe plus complexe}

Voyons les exemples. Nous indiquons dans les exemples la version suédoise suivie, entre parenthèses, de notre traduction mot à mot, qu'il sera parfois intéressant de connaître. Les exemples mentionnent aussi la version française, après la barre oblique, ainsi que le titre en français. Dans la bibliographie, tous les titres et dates de publications sont disponibles. Voyons l'exemple (1). On voit que le texte suédois contient deux phrases. Elles sont reliées entre elles en français par une simple virgule pour former une seule phrase plus longue. Le sujet est aussi omis dans la version française 〈verse〉 〈prépare〉. De plus, la coordination 〈och〉 (et) qui apparaît dans la deuxième phrase suédoise a été traduite par un marqueur temporel explicite spuis〉, ce qui est relativement courant dans les traductions:

(1) Tant Alma ställer fram ett stort fat med bullar på köksbordet. Hon häller upp saft i fyra glas och kokar kaffe åt sig själv och tant Märta (Madame Alma pose un grand plat de brioches sur la table de la cuisine. Elle sert du sirop dans quatre verres et fait du café pour elle-même et madame Märta) / Tante Alma pose une corbeille remplie de brioches sur la table de la cuisine, verse du jus de fruits dans quatre verres puis prépare du café pour tante Marta et pour ellemême. Thor, Annika (2003): Une île trop loin.

Dans l'exemple (2) les phrases simples du texte suédois sont reliées entre elles en français et la syntaxe est en quelque sorte rétablie: la proposition qui commence par le pronom interrogatif 〈hur〉 (comment) est incluse dans une proposition plus longue 〈qu'à plusieurs reprises...>. Il y a une phrase en français au lieu de deux en suédois. De plus, cela change un peu le sens puisque l'enfant en suédois répète 〈hans pappa〉 (son papa) alors qu'en français on peut avoir du mal à cerner l'antécédent de 〈il» et de 〈ses〉.

(2) Det var något som hans pappa hade berättat. Hur han flera gånger hade kommit gående i sin nattskjorta, ut i rummet där hans pappa satt och lyssnade på radion eller bläddrade i några av sina gamla sjökort. (C'était quelque chose que son papa avait raconté. Qu'il était venu plusieurs fois en chemise de nuit, dans la pièce où son papa était assis et écoutait la radio et feuilletait quelques-unes de ses vieilles cartes marines) Son père lui a raconté qu'à plusieurs reprises il 
était apparu en chemise de nuit dans la pièce où il se retirait pour écouter la radio ou feuilleter ses vieilles cartes marines. Mankell, Henning (1990): La société secrète.

\subsection{Un vocabulaire plus complexe}

Dans l'exemple (3), en suédois l'auteur emploie 〈inte farligt〉 (pas dangereux) lorsque le bonhomme Pettson décrit un blaireau à son chaton qui en a eu très peur. Le chaton est comme son petit enfant. En français c'est remplacé par 〈inoffensif), variante énonciative plutôt caractéristique de l'écrit que de l'oral et sans doute peu habituelle pour un jeune enfant. L'auteur de Pettson choisit souvent de nier un adjectif connu du lectorat enfantin (par le préfixe suédois o-) plutôt que d'en employer un plus compliqué.

(3) Inte då, sa Pettson. Det där var den gamle grävlingen. Den äter inte katter. Om du inte retar den för mycket, så är den inte farlig (Mais non, dit Pettson. Ça c'était le vieux blaireau. Il ne mange pas les chats. Si tu ne le taquines pas trop, il n'est pas dangereux) / Mais non, dit Pettson, ce n'est pas dangereux. Ce vieux blaireau est inoffensif. Nordqvist, Sven (2005): Le jour où Picpus a disparu.

\subsection{Une narration plus complexe}

Les petits hippopotames dans l'exemple (4) obtiennent enfin l'autorisation d'aller sauter dans l'eau du haut de la grande falaise. Dans la version française publiée, le locuteur n'est pas nommé, ce qui complique la compréhension par les petits enfants, à qui le livre est destiné (de 3 à 5 ans). En suédois, le locuteur est nommé, bien que postposé «säger de till varann〉 (se disent-ils). En français l'on peut aussi se demander qui est «il», non nommé, ni dans le cotexte, ni sur l'illustration:

(4) Bäst att gå genast, säger de till varann (Mieux vaut y aller tout de suite, se disent-ils) / Dépêchons-nous d'y aller avant qu'il ne change d'avis. Landström, Lena (2001): Trois petits hippopotames.

Pour conclure, il faut dire que ces trois modifications stylistiques sont souvent en jeu dans la traduction d'autres genres. Il existe des différences importantes entre ces deux langues, au niveau sémantico-lexical et stylistique. Or dans les livres pour enfants, certaines structures - comme les phrases courtes ou les répétitions, les termes simples, ont comme fonction d'installer une perspective enfantine, respectant la logique propre aux enfants, du moins selon les auteurs.

Nous allons maintenant au cœur de notre exposé qui sera développé en deux temps: l'oralité écrite et plus spécialement dans les livres pour enfants et ensuite la traduction. 


\section{La représentation de l'oral à l'écrit dans les traductions en français}

Tout d'abord il peut être intéressant de remarquer que le suédois a formalisé l'oral à l'écrit, pour certains mots courts et fréquents, dont certains sont donnés dans l'exemple (5).

(5) $\operatorname{mig}=\operatorname{mej}(\mathrm{moi}), \operatorname{dig}=\operatorname{dej}($ toi), $\mathrm{de}=\mathrm{dom}$ (ils), någon $=$ nån (quelqu'un), något $=$ nått (quelque chose), sedan $=$ sen (ensuite)

Les pronoms et l'adverbe temporel de l'exemple (5) ont deux formes écrites, la première plus formelle et l'autre informelle. La variante informelle est construite en essayant d'imiter la prononciation des mots dans le suédois standard. La variante informelle est utilisée surtout dans l'écrit spontané et dans la littérature pour rendre la narration et les personnages plus vrais. ${ }^{3}$ Les variantes informelles sont donc très fréquentes dans la littérature enfantine. Comme ces formes n'existent le plus souvent pas en français, elles ne sont traduisibles que par compensation, de la même façon que - exemple bien connu, les formes anglaises 〈I'm〉 ou 〈you're〉, etc. Une compensation possible, et qui est relativement fréquente dans, par exemple, la traduction de Tsatsiki de Moni Nilsson-Brännström, serait ici l'usage des formes 〈t'as〉, 〈t'es〉 qui, elles, n'ont pas d'équivalent en suédois. A ces mots se rajoute une liste ouverte de formes créées par les auteurs, pour lesquelles il n'existe pas de forme écrite standardisée. Dans l'exemple (6) on voit l'emploi de 〈dom», pronom personnel de troisième personne du pluriel, à la place de la variante formelle 〈de〉. 〈Dom〉 appartient au même registre de langue que l'adjectif suivant 〈heläckliga〉 (dégueulasses):

(6) Har du sett dom sa jag. Dom är heläckliga (Tu les as vus, dis-je. Ils sont vraiment dégueulasses) / Tu les as vus? Ils sont vraiment dégueulasses. Thor, Annika (1999): Le jeu de la vérité.

Dans l'exemple (7), il y a 〈middan〉 (le dîner) à la place de 〈middagen〉. On a une réduction de l'article défini postposé 〈-en〉 en 〈-n〉 et le 〈g〉 final tombe. On a aussi 〈sen〉 à la place de 〈sedan〉 (ensuite).

(7) Var har du varit?!! Middan har kallnat för länge sen! (Où as-tu été? Le dîner a refroidi depuis longtemps!) / Où étais-tu passée? Le dîner est tout froid maintenant. Höglund, Anna (1998): Lola s'en va.

En outre, comme l'a montré Lagerholm (1999) en analysant des protocoles du parlement, des articles de presse, des récits de voyage, etc. de 1800 à 1997 à partir d'une adaptation du modèle de Biber (notamment 1988), l'écrit se rapproche de plus en plus de l'oral dans plusieurs genres de textes en suédois.

Dans la littérature pour enfant, l'oralité a longtemps été en Suède un trait stylistique récurent, de sorte qu'elle n'est pas jugée par les spécialistes de littérature enfantine comme

3 Cette hétéroglossie, selon le terme de Bakhtin (1981: 301-302), ou mélange de styles et des niveaux de langues, chez plusieurs ou un seul locuteur, risque toujours [en général] d'être neutralisée dans la traduction (Pietilä 2003: 15), comme nous le verrons. 
quelque chose de particulièrement original: il y a souvent des imitations de langage des jeunes ou des gros mots dans les discours directs, sans que l'évolution n'aille vers une augmentation de ce phénomène.

Mais, les mêmes spécialistes en France, les éditeurs et les traducteurs réagissent à cette oralité. ${ }^{4} \mathrm{La}$ politique éditoriale française visant à n'écrire que du 〈bon〉 ou $\mathrm{du}$ 〈beau〉 français sauf éventuellement dans les discours directs est toujours d'actualité. Il semble encore régner en France une conception selon laquelle les termes 〈parlés〉 ne s'écrivent pas, ni dans les discours directs ni dans les narrations. Pourtant certains auteurs en France veulent représenter l'oral à l'écrit de façon plus réaliste, comme nous le verrons ci-dessous, dans des exemples tirés de notre corpus français contemporain de contrôle. On retrouve quelques traces (plus ou moins acceptées selon le genre de texte) de l'oral à l'écrit, par exemple la ponctuation qui peut exprimer l'exclamation ou l'ironie. Parfois on trouve ce que Claire Blanche-Benveniste appelle des «trucages d'orthographe» (voir l'exemple 8) (1997: 26) ou «orthographe estropiée» qui sont des symboles de français 〈populaire» voir d'illettrisme. L'exemple 8, qui est tiré de notre corpus français de contrôle est intéressant, non seulement puisqu'il montre la retranscription graphique de «nan〉 à la place de «non〉, l'emploi de l'apostrophe qui est aussi typique de la représentation écrite de l'oral en français, mais aussi le manque de négation (au lieu de 〈tu n’y vas pas〉, 〈je n’ai pas finì, 〈ça ne fait rien〉) et la syntaxe inversée dans l'incise (au lieu de 〈lui dis-je〉).

(8) - T'y vas pas? je lui dis - Nan, j'ai pas fini! - Ça fait rien, tu peux venir après. - Nan, j'te dis. - Tu veux que j'te rate ton jeu de piste ou quoi? Vignal, Hélène (2007): Bière grenadine.

Un deuxième exemple éloquent à ce sujet est l'exemple (9). On voit les formes 〈parlées〉 et 〈familières〉, comme 〈meufs, con〉 puis un changement de registre dans les deux derniers tours de parole, par exemple «ne détourne pas la conversation〉 (ce qui est normal puisque chaque être humain qui parle maîtrise plusieurs registres), mais aussi la voix de l'auteur dans les incises au passé simple.

(9) - Y a pas une fille! s'écria-t-il, épouvanté. - La faute à qui? cria à son tour Corentin. Aller en boîte, c'est con. Draguer dans la rue, c'est con. Se faire les meufs des autres, c'est con. - Ça se dit encore, «meuf»? - Ne détourne pas la conversation. Murail, Marie-Aude (2004): Simple.

L'oralité existe donc aussi bien dans la littérature pour enfants suédoise que française même si elle se manifeste bien évidemment de différentes manières. Comme le rappelle Liisa Tiittula (2001: 8-10), il ne s'agit que d'une illusion, puisque l'écrit ne pourra jamais qu'imiter l'oral sans pouvoir copier: intonation, ryhtme, pauses, etc. Pourtant dans les traductions l'oralité disparaît ou elle est édulcorée. Ceci a déjà été remarqué en ce qui concerne la traduction du suédois au finnois par Pia Pietilä (2003) qui a étudié la traduction d'une bande dessinée suédoise particulièrement oralisée: le traducteur utilise le plus souvent des formes considérées comme neutres du point de vue stylistique et en tous cas moins de marqueurs orthographiques d'oralité. De même, Birgitta Englund-Dimittrova (1997; 2001) souligne la standardisation des traductions. Ce processus permet une

4 Le désir d'étudier «les spécificités de l'oral [rendues] à l'aide de la langue écrite» est lui-même assez nouveau (cf. Ballard 2001). 
traduction toute en douceur, et pour le traducteur, et pour le lecteur, puisque ce sont surtout les marqueurs lexicaux d'oralité qui se retrouvent dans le texte traduit, au détriment des marqueurs orthographiques. Voyons maintenant deux aspects de la représentation de l'oral à l'écrit dans notre sous-corpus: les 〈gros mots〉 et le parler enfantin.

\subsection{Les gros mots}

Lars-Gunnar Andersson (1985) distingue trois genres différents, pour la langue suédoise: (a) une variante oralisée d'une expression formelle, (b) un terme formel grossier en soi et (c) des termes argotiques ou 〈parlés〉 dont le contenu est grossier. Il souligne que les gros mots sont toujours plus fortement ressentis à l'écrit qu'à l'oral. Dans les discours directs des injures et des gros mots sont utilisés sans que cela ne soit spécialement remarqué en Suède. Bien sûr certains réagissent et voudraient encourager l'emploi d'un langage châtié dans la littérature pour enfants. Dans les traductions françaises, ces gros mots ont tendance à disparaître ou à être adaptés. La romancière française Marie-Aude Murail remarquait en 2005 que les maisons d'édition poussent encore de nos jours à ne pas employer de «gros mots〉. Elle-même reçoit régulièrement des lettres de parents indignés si elle emploie un terme jugé déplacé. Alors que, citons Marie-Aude Murail:

Mes personnages veulent rester crédibles. J'explique régulièrement aux enfants que Gavroche n'appelle pas les gendarmes des 〈keufs〉 parce qu'il n'est pas du 93, mais il les surnomme des 〈cognes», ce qui correspond à l'argot de son époque. C'est, pour Victor Hugo, une question de vraisemblance. Je ne suis pas hors champ littéraire quand j'emploie de l'argot ou des 〈gros mots>, bien au contraire, j'appartiens à une tradition.

Dans les exemples $(10,11,12)$ on voit que les locuteurs suédois emploient de très gros mots, édulcorés dans la version française. Dans l'exemple (10) c'est une réplique violente et méchante, en plus d'être grossière. Il est intéressant de voir que l'auteur n'utilise pas la version oralisée du juron 〈vad fan〉, qui est pourtant courante (〈vafan〉).

(10) Vad fan gör du, ditt jävla offer! sa hon. Gå och sätt dig för helvete (Putain qu'est-ce que tu fous, sale victime! dit-elle. Va t'asseoir, putain!) / Qu'est-ce que tu fous, espèce de minable! Va t’asseoir, merde! Thor, Annika (1999): Le jeu de la vérité.

Dans (11) la traductrice a compensé l'atténuation par un commentaire sur la couleur du visage du jeune garçon, qui n'est pas signalée dans la version originale. De plus, la négation est entière «ne...pas〉 alors que la première partie «ne〉 en est souvent omise pour marquer l'oralité.

(11) «Men för fan!» utbrister han. (Mais putain! s'exclame-t-il) / Mais vous ne comprenez pas, s'écrie-t-il, rouge d'excitation. Nordin, Sofia (2003): Seuls!

Dans (12) le garçon emploie textuellement 〈j’ai cru que j'allais me chier dessus〉 qui ne passe pas en français. D'après les traductrices que nous avons rencontrées, les textes suédois font beaucoup plus référence à la scatologie que ce dont on a l'habitude en France. 
Ces traductrices suppriment et essaient de compenser les allusions et expressions scatologiques.

(12) «Jag trodde jag skulle skita på mig», säger han. «Inte för att jag blev rädd...» börjar han hastigt att lägga till, som av gammal vana, men ändrar sig sedan «Jo, det är klart att jag blev rädd. Som fan». («Je croyais que j'allais me chier dessus», dit-il. «Pas parce que j'ai eu peur...» commence-t-il rapidement à ajouter, par habitude, puis il change d'avis «si, c'est clair que j'ai eu peur. Les boules!») / - Un élan! pouffe-t-il [...] J'ai cru que j'allais faire dans mon pantalon. Pas parce que j'ai eu peur ... Enfin si, bien sûr que j'ai eu peur. La peur de ma vie. Nordin, Sofia (2003): Seuls!

\subsection{Le parler 〈enfantin>}

La littérature suédoise est réputée pour rendre et respecter le point de vue de l'enfant. Pour ne parler ici que du niveau linguistique, voyons quelques exemples de sparler enfantin〉. L'exemple (13) est tiré de la célèbre série des Max, en France Mini Bill puis Tom. C'est traduit en français par des phrases simples mais qui ne rendent pas la version suédoise en langage bébé. Dans les premières éditions, le texte était même volontairement traduit en langage 〈adulte〉:

(13) Titta Max! Titta Max boll! Max leker bollen. Kommer kissen. Jam Jam. Kissen tar bollen (Regarde Max! Regarde ballon Max! Max joue le ballon. Arrive le minou. Miaou. Miaou. Le minou prend le ballon) / Tom a trouvé quelque chose. Oh, un ballon. Tom joue avec le ballon. Le chaton arrive. MIAOU. Le chaton prend le ballon. Lindgren, Barbro / Eriksson, Eva (1989): Le ballon de Tom.

L'exemple (14) montre une forme syntaxiquement non normée, un emploi du pronom personnel sujet 〈han〉 à la place du pronom personnel complément 〈honom». Ce n'est pas accepté à l'oral en suédois non plus mais c'est une forme que l'on retrouve souvent dans l'oral représenté à l'écrit dans la littérature enfantine. On voit que dans la deuxième phrase l'auteur emploie le pronom personnel complément d'objet correct. C'est une façon de montrer les différents registres. Dans la traduction en français, le langage enfantin disparaît puisqu'il est difficile à rendre en français comme vous le voyez dans notre traduction mot à mot. L'adjectif 〈ball〉 traduit par 〈costaud〉 met l'accent sur la force et la brutalité des cochons, alors qu'en suédois on insistait plutôt sur leur côté à la mode, 〈cool〉 et non sur leur force.

(14) - Vi slår han på trynet, säger de balla grisarna. [...] Den ballaste slår honom på trynet så nappen flygger ut. (On tape il sur le groin, disent les supers cochons. Le plus super/cool le tape sur le groin si fort que la tétine s'envole) / - Si on lui donnait un coup de poing sur le nez? disent les cochons costauds [...]. Le plus costaud des trois lui donne un coup de poing dans le nez. Lindgren, Barbro / Landström, Olof (2005): La tototte.

L'exemple (15) montre un changement radical du niveau de style, passant d'un mot typiquement enfantin 〈dösäkert〉 à 〈à toute épreuve〉. Le préfixe 〈dö-〉 qui signifie en fait 
〈mort〉 est utilisé en suédois pour intensifier le mot préfixé. C'est une jeune fille d'une douzaine d'années qui parle.

(15) [Jag menar att jag har den där silverkannan!] På ett dösäkert ställe. ([je veux dire que c'est moi qui ai ce pot en argent!] Dans un endroit super sûr) / Je veux dire que c'est moi qui ai le pot en argent! Dans une cachette à toute épreuve. Johansson, Elsie (2003): Du courrier pour le chat.

En guise de conclusion, on peut dire que cette analyse pilote nous a permis de voir que la représentation de l'oral est un moyen très souvent utilisé en suédois et en français pour transmettre une perspective enfantine, de sorte que l'enfant lecteur ou auditeur peut, dans l'idéal, se reconnaître. De même, les adultes lecteurs reconnaissent des enfants d'après l'idée qu'ils s'en font.

Or, lors de la traduction du suédois vers le français on a une tendance à changer de registre qui pert d'un côté son oralité et de l'autre la tonalité familière et, a fortiori, la perspective de l'enfant. On pense à trois explications possibles: les différences linguistiques structurelles, les normes stylistiques différentes et les attentes des lecteurs dans les deux cultures quant à la littérature pour enfants. Comme nous l'avons mentionné en introduction, le lectorat adulte suédois a souvent une vue très 〈enfantine〉 de l'enfance et des enfants et tolère, voire demande, une perspective enfantine poussée en littérature.

Nos prochaines recherches nous permettront de trouver le poids relatif de ces trois explications.

\section{Corpus}

Fastier, Yann (2000): Savoir-vivre. Paris: L'Atelier du Poisson Soluble.

Hoestlandt, Jo (2001): La dent d'Ève. Paris: Actes sud Junior.

Hugo, Hector (1998): Aubagne la galère. Paris: Mini Syros Polar.

Höglund, Anna (1997): Mina går sin väg. Stockholm: Alfabeta; (1998): Lola s'en va. Paris: Seuil.

Landström, Lena (2000): Småflodhästarnas äventyr. Stockholm: Rabén \& Sjögren; (2001): Trois petits hippopotames. Paris: L'école des loisirs.

Lindgren, Barbro / Eriksson, Eva (1982): Max boll. Stockholm: Rabén \& Sjögren; (1989): Le ballon de Tom. Paris: L'école des loisirs.

Lindgren, Barbro / Landström, Olof (2001): Jamen Benny. Stockholm: Rabén \& Sjögren; (2005): La tototte. Paris: L'école des loisirs.

Mankell, Henning (1990): Hunden som sprang mot en stjärna. Stockholm: Rabén \& Sjögren; (1998): La société secrète. Paris: Flammarion.

Nilsson-Brännström, Moni (1995): Tsatsiki och morsan. Natur och kultur; (2004): Tsatsiki. Paris: Flammarion.

Murail, Marie-Aude (2004): Simple. Paris: L'école des loisirs.

Nordin, Sofia (2003): Äventyrsveckan. Stockholm: Andersson \& Lindgren; (2005): Seuls! Paris: Casterman.

Nordqvist, Sven (2001): När Findus var liten och försvann. Stockholm: Opal; (2005): Le jour où Picpus a disparu. Paris: Autrement.

Rossignol, Isabelle (2007): Mamie Colette \& Co. Paris: L'école des loisirs. 
Thor, Annika (1996): En ö i havet. Stockholm: Bonnier Carlsen; (2003): Une île trop loin. Paris: Thierry Magnier.

- (1997): Sanning eller konsekvens. Stockholm: Bonnier Carlsen; (1999): Le jeu de la vérité. Paris: Casterman.

Vignal, Hélène (2007): Bière grenadine. Paris: éditions du Rouergue.

\section{Bibliographie}

Andersson, Lars-Gunnar (1985): Fult språk. Stockholm: Carlsson.

Bakthin, Mikhail (1981): Discourse in the novel. In: The Dialogic Imagination. Four essays. Austin: University of Texas Press, 301-331.

Ballard, Michel (ed.) (2001): Oralité et traduction. Arras: Artois Presses Université.

Biber, Douglas (1988): Variation across speech and writing. Cambridge: Cambridge University Press.

Blanche-Benveniste, Claire (1997): Approches de la langue parlée en français. Paris: Ophrys.

Englund-Dimitrova, Birgitta (1997): Translation of Dialect in Fictional Prose - Vilhem Moberg in Russian and English as a Case Point. In: Norm, Variation, and Change in Language Proceeding of the Centaury Meeting of the Nyfilologiska Sällskapet Nedre Manilla 22-23 March 1996. Stockholm: Almqvist \& Wiksell, 49-65.

- (2001): En smålänning $i$ engelsk och fransk skepnad - om översättningen av dialekt $i$ skönlitteratur. In: Folkmålsstudier 40. Helsingfors: Universitetstryckeri, 9-27.

Kåreland, Lena (1998): 1990-talets svenska bilderbok. Några exempel. In: Hallberg, Kristin (ed.): Läs mig. Sluka mig! En bok om barnböcker. Stockholm, 277-298.

-(2001): Möte med barnboken: linjer och utveckling $i$ svensk barn-och ungdomslitteratur. Stockholm: Natur \& Kultur.

Lagerholm, Per (1999): Talspråk i skrift: om muntlighetens utveckling i svensk sakprosa 1800-1997. Lund: Lundastudier.

Lindgren, Charlotte / Andersson, Carina / Renaud, Catherine (2007): La traduction des livres pour enfants suédois en français: choix et transformation. In: La Revue des livres pour enfants 234, 8793.

Murail, Marie-Aude (2005): Dialogue avec Nadège. In: Nièvres-Chevrel, Isabelle (ed.): Littérature de Jeunesse, incertaines frontières. Paris: Gallimard Jeunesse.

Nikolajeva, Maria (2004): Till otrohetens försvar: om att svika texten till förmån för barnläsaren. In: Barnboken 27-1.

Oittinen, Riitta (2000): Translating for children. New York: Garland.

Pietilä, Pia (2003): Borta bra men hemma bäst? En enkätundersökning av tre finska översättningar av en tecknad serie. Pro-gradu avhandling. Helsingfors: Institution för nordiska språk och nordisk litteratur.

Renaud, Catherine / Andersson, Carina / Lindgren, Charlotte (2007): L'image dans la traduction de livres pour enfants: défi ou soutien. Réflexions à partir de la traduction en français de quelques livres pour enfants suédois. In: La Revue des livres pour enfants 234, 94-101.

Rhedin, Ulla (2004): Bilderbokens hemligheter. Stockholm: Alfabeta.

Tiittula, Liisa (2001): Puhuttu kieli ja sen illuusio kirjallisuudessa. Kuinka puhe kääntyy tekstiksi? In: Kääntäjä-Översättaren $\mathrm{Nr}$ 6. Helsinki: SKTL, 8-10.

Toury, Gideon (1995): Descriptive Translation Studies and Beyond. Amsterdam: John Benjamins publishing company.

Westin, Boel (1998): La litterature enfantine en Suède. Paris: Institut suédois. 
